\title{
On power allocation for generalized cyclic-prefix based channel-equalizers
}

\author{
P. P. Vaidyanathan and Bojan Vrcelj \\ Dept. Electrical Engineering, California Institute of Technology \\ Pasadena, CA 91125, USA \\ ppvnath@systems.caltech.eduｂojan@systems.caltech.edu
}

\begin{abstract}
The cyclic prefix system is commonly employed for channel equalization in discrete multitone systems. The system allows one to perform bit and power allocation in the subbands of the channel. In the DMT system, the input symbol stream, typically binary, is parsed into several substreams which are then communicated over different subbands of the channel. In this paper we emphasize that the cyclic prefix can actually be used in a broader setting. For example, if we have to transmit a simple symbol stream belonging to a PAM constellation we can use the cyclic prefix directly on this stream. More generally, the idea is applicable to the problem of compensating any linear distortion with additive noise. We derive the optimal power allocation formula for the case of a nonflat channel with possibly colored noise.
\end{abstract}

\section{INTRODUCTION}

The cyclic prefix system is commonly employed for channel equalization in discrete multitone systems [7]. These systems are used for communication on twisted pair channels in telephone cables [1], [8]. The system allows one to perform bit and power allocation in the subbands of the channel. The implementation is based on the DFT, and is therefore very efficient. In the DMT system, the input symbol stream, typically binary, is parsed into several substreams which are then communicated over different subbands of the channel. The substreams typically have different bit rates representing constellations of different sizes, and there exist standard techniques for optimal power allocation across the subchannels [8].

In this paper we emphasize that the cyclic prefix can actually be used in a broader setting (Sec. II, III). For example, if we have to transmit a simple symbol stream belonging to a PAM constellation we can use the cyclic prefix directly on this stream. The problem of power allocation becomes very different in this case as explained in Sec. IV. In Sec. V we derive the optimal power allocation formula for the case of a nonflat channel with possibly colored noise. Examples are presented in Sec. VI comparing the optimal cyclic prefix equalizer with the traditional zero-forcing equalizer. It will be assumed throughout the paper that the channel is an FIR system

$$
C(z)=\sum_{n=0}^{L} c(n) z^{-n}
$$

\footnotetext{
${ }^{1}$ Work supported in part by the ONR grant N00014-99-11002, USA.
}

with additive noise $e(n)$ as shown in Fig. 1. This assumption is often justified by the use of a time domain equalizer prior to using the cyclic prefix system [4].

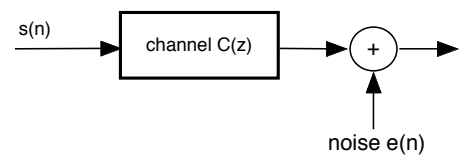

Figure 1. A simple FIR channel.

\section{CYCLIC-PREFIX REVIEW}

In this section we place the cyclic prefix system in a broader perspective so that its wider applicability becomes clear. Figure 2(a) shows the input stream divided into blocks of length $M$. The $L$ symbols at the end of each block (where $L=$ channel order) are copied into the beginning of that block, to form the cyclic prefix (thin lines in Fig. 2(c)). This evidently assumes $L \leq M$. For a given symbol rate, the cyclic-prefix reduces the spacing between samples. The factor $\gamma=(M+L) / M$ represents the excess bandwidth required for this. By making $M$ sufficiently large we can reduce $\gamma$ (at the expense of latency in symbol detection).

Let $\mathbf{s}(n)$ denote the vector of $M$ input symbols $s(n)$ in the $m$ th block, and let $\mathbf{y}(n)$ be the vector of last $M$ output symbols in the $m$ th block:

$$
\begin{gathered}
\mathbf{s}(m)=\left[\begin{array}{llll}
s(m M) & s(m M+1) & \ldots & s(m M+M-1)
\end{array}\right]^{T} \\
\mathbf{y}(m)=\left[\begin{array}{llll}
y\left(J_{m}\right) & y\left(J_{m}+1\right) & \ldots & y\left(J_{m}+M-1\right)
\end{array}\right]^{T}
\end{gathered}
$$

with $J_{m}=m(L+M)+L$. If we make the stronger requirement that $L<M$, then in absence of noise we can show that

$$
\mathbf{y}(m)=\mathbf{C s}(m)
$$

where $\mathbf{C}$ is circulant with the elements of the top row coming from the channel impulse response $c(n)$. For example when $L=3$ and $M=6$,

$$
\mathbf{C}=\left(\begin{array}{cccccc}
c(0) & 0 & 0 & c(3) & c(2) & c(1) \\
c(1) & c(0) & 0 & 0 & c(3) & c(2) \\
c(2) & c(1) & c(0) & 0 & 0 & c(3) \\
c(3) & c(2) & c(1) & c(0) & 0 & 0 \\
0 & c(3) & c(2) & c(1) & c(0) & 0 \\
0 & 0 & c(3) & c(2) & c(1) & c(0)
\end{array}\right)
$$



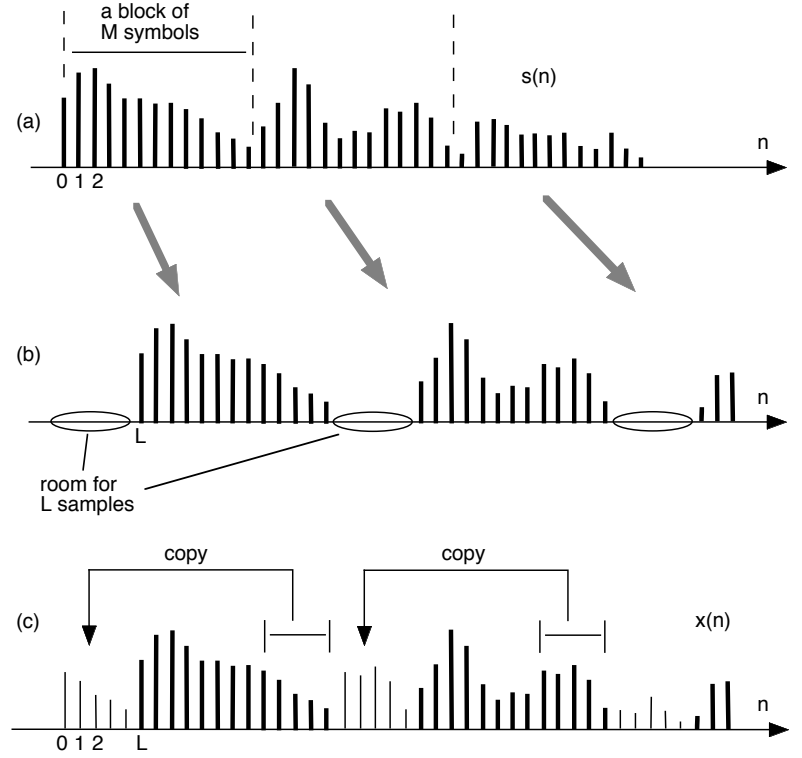

(d)

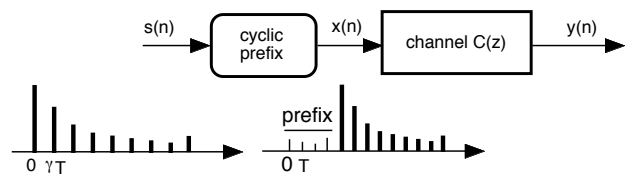

Figure 2. (a) Input symbol stream, (b)-(c) explanation of how cyclic prefix is inserted, and (d) block diagram.

If the channel is known, we can perform the equalization by inverting (2) assuming $\mathbf{C}$ is nonsingular. Any circulant can be diagonalized with the DFT matrix [6]. More specifically $\mathbf{C}=\mathbf{W}^{-1} \boldsymbol{\Lambda}_{c} \mathbf{W}$ where $\mathbf{W}$ is the $M \times M$ DFT matrix and

$$
\boldsymbol{\Lambda}_{c}=\operatorname{diag}\{C[0], C[1], \ldots C[M-1]\}
$$

where $^{2} C[k]=\sum_{n=0}^{L} c(n) W^{n k}=M$-point DFT of $c(n)$. Thus the implementation of the communication system with cyclic prefix can be represented as shown in Fig. 3. The box labelled "blocking" is a serial to parallel converter (and "unblocking" converts from parallel to serial). The diagonal elements of $\left[\boldsymbol{\Lambda}_{c}\right]^{-1}$ are $1 / C[k]$, and can be regarded as a set of DFT-domain equalizers.

\section{SCHEMATIC REPRESENTATION}

Since $\mathbf{y}(m)=\mathbf{C s}(m)$ according to Eq. (2), we can draw a schematic version of Fig. 3 as shown in Fig. 4(a). As $\mathbf{W}^{-1}$ is the inverse of $\boldsymbol{\Lambda}_{c}^{-1} \mathbf{W C}$, we can redraw the system as in Fig. 4(b). In the first version the receiver has all the complexity whereas in the second version the IDFT is done at the transmitter, as in traditional DMT systems. We can choose $M$ to be a power of two and implement $\mathbf{W}$ and $\mathbf{W}^{-1}$ using radix-2 FFT.

In the next section we take channel noise into account. If $C(z)$ has zeros close to the unit circle, then some of the

\footnotetext{
${ }^{2}$ We use the standard notation $W=e^{-j 2 \pi / M}$.
}

$C[k]$ could be very small, and $\mathbf{C}$ becomes ill condition, thus amplifying channel noise severely. Note that the traditional zero-forcing equalizer $1 / C(z)$ is unstable if the channel has zeros in $|z| \geq 1$. One advantage of the cyclic prefix method is that the channel does not have to have minimum phase. It is sufficient that $C\left(e^{j \omega}\right)$ be non zero at the DFT frequencies (i.e., $C[k] \neq 0$ for any $k$ ).

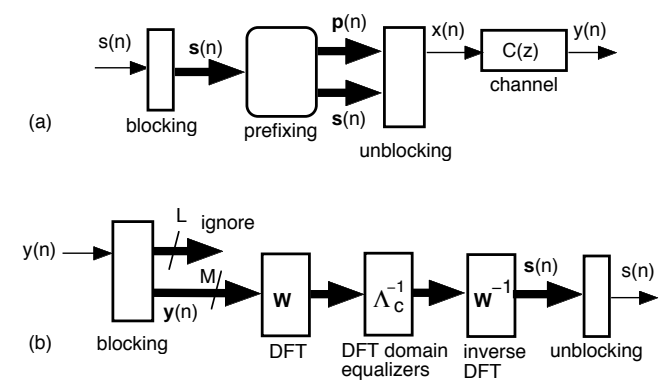

Figure 3. Block diagram description of the communication system based on cyclic prefix. (a) Transmitter, and (b) receiver.
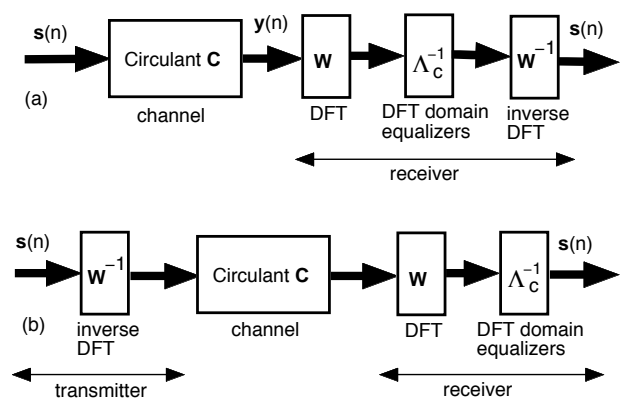

Figure 4. (a) A simplified schematic of the cyclic prefix system, and (b) a practically useful rearrangement similar to the conventional DMT system.

\section{PREMULTPLIERS AND DETECTOR SNR}

Consider Fig. 5(a) which shows the system of Fig. 4(b) with two modifications: first there is a unitary matrix $\mathbf{U}$ at the receiver, and its inverse $\mathbf{U}^{\dagger}$ (transpose conjugate) at the transmitter. Second, there is a diagonal matrix $\boldsymbol{\Lambda}_{p}$. Before explaining the purpose of these, notice first that as far as the signal path is concerned, the matrices $\mathbf{U}$ and $\mathbf{U}^{\dagger}$ cancel. So do $\boldsymbol{\Lambda}_{p}$ and $\boldsymbol{\Lambda}_{p}^{-1}$.

The matrices $\boldsymbol{\Lambda}_{p}^{-1}$ and $\mathbf{U}$ affect the way the receiver processes channel noise. They can be chosen to maximize the signal to noise ratio at the detector. The matrix $\boldsymbol{\Lambda}_{p}$ also affects the distribution of signal power on the channel. We will see that the diagonal elements $p_{k}$ of the diagonal matrix $\boldsymbol{\Lambda}_{p}$ allow us to allocate power in various subbands of the channel, based on the channel gain and noise in subbands. Before proceeding to the details we first explain the purpose of the unitary matrix $\mathbf{U}$.

If $e(n)$ is wide sense stationary (WSS) the blocked version $\mathbf{e}(n)$ has a Toeplitz autocorrelation matrix $\mathbf{R}_{e e}$. However, the received noise component $\mathbf{g}(n)$ does not have a 
Toeplitz autocorrelation (even the diagonal elements are not equal in general). The unblocked noise $g(n)$ (Fig. $5(\mathrm{~b}))$ therefore has a variance that is periodic in $n$ with period $M$. The SNR at the receiver then becomes a function of time, and so does the error probability in symbol detection. Careful choice of the unitary matrix $\mathbf{U}$ avoids this problem as explained next. The noise vector $\mathbf{g}(n)$ has autocorrelation $\mathbf{T} \mathbf{R}_{e e} \mathbf{T}^{\dagger}$, where $\mathbf{T}=\mathbf{U} \boldsymbol{\Lambda}_{p}^{-1} \boldsymbol{\Lambda}_{e} \mathbf{W}$ and $\mathbf{R}_{e e}=E\left[\mathbf{e}(n) \mathbf{e}^{\dagger}(n)\right]$. Thus

$$
\mathbf{R}_{g g}=\mathbf{U} \boldsymbol{\Lambda}_{p}^{-1} \boldsymbol{\Lambda}_{e} \mathbf{W} \mathbf{R}_{e e} \mathbf{W}^{\dagger} \boldsymbol{\Lambda}_{e}^{\dagger} \boldsymbol{\Lambda}_{p}^{-\dagger} \mathbf{U}^{\dagger}=\mathbf{U} \mathbf{Z} \mathbf{U}^{\dagger}
$$

where $\mathbf{Z}=\boldsymbol{\Lambda}_{p}^{-1} \boldsymbol{\Lambda}_{e} \mathbf{W} \mathbf{R}_{e e} \mathbf{W}^{\dagger} \boldsymbol{\Lambda}_{e}^{\dagger} \boldsymbol{\Lambda}_{p}^{-\dagger}$. Given any positive definite $\mathbf{Z}$, we can always find a unitary $\mathbf{U}$ such that $\mathbf{U Z U}^{\dagger}$ has identical diagonal elements. This result was first introduced in the signal processing community by Mullis and Roberts in 1976, and an algorithm can be found in [5]. For the special case of white noise $\mathbf{Z}$ is diagonal, and we can choose $\mathbf{U}=\sqrt{M} \mathbf{W}^{-1}$ so that $\mathbf{U Z U ^ { \dagger }}$ is circulant, and in particular has identical diagonal elements.
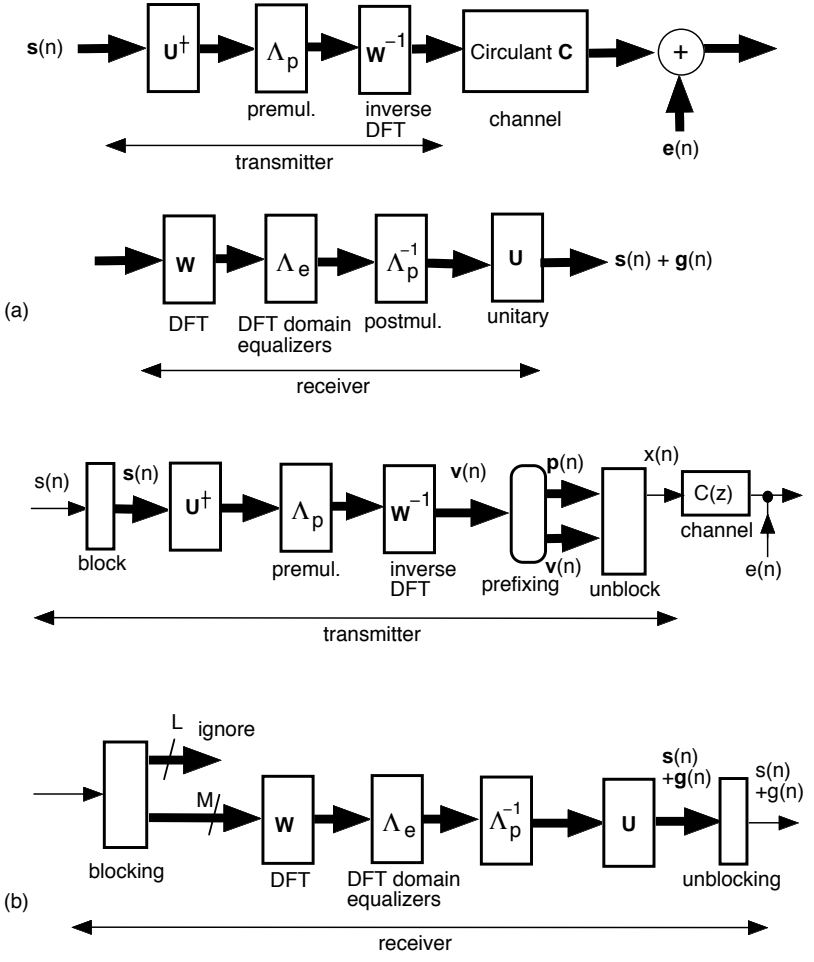

Figure 5. (a) The cyclic prefix system with pre and postmultipliers, and a unitary matrix $\mathbf{U}$ for postprocessing. (b) Schematic showing more details.

IV.1. Making The Noise Variance Time Invariant Our goal is to choose $\boldsymbol{\Lambda}_{p}$ such that the SNR at the detector is maximized for fixed power input to the channel. For any unitary $\mathbf{U}$, the variance $\sigma_{g}^{2}$ averaged over $M$ successive samples can be calculated as

$$
\sigma_{g}^{2}=\frac{1}{M} \operatorname{Tr} \mathbf{R}_{g g}=\frac{1}{M} \operatorname{Tr} \mathbf{Z}=\frac{1}{M} \operatorname{Tr}\left(\boldsymbol{\Lambda} \mathbf{W} \mathbf{R}_{e e} \mathbf{W}^{\dagger}\right)
$$

where $\boldsymbol{\Lambda}$ is a diagonal matrix with diagonal elements $\lambda_{i}=1 /\left|C[i] p_{i}\right|^{2}$. Note that $\sigma_{g}^{2}$ is independent of $\mathbf{U}$. In fact we will see that the optimal choice of $\boldsymbol{\Lambda}_{p}$ itself does not depend on $\mathbf{U}$. Once $\boldsymbol{\Lambda}_{p}$ is computed, $\mathbf{Z}$ can be found and $\mathbf{U}$ identified such that $E\left[|g(n)|^{2}\right]=\sigma_{g}^{2}$ for all $n$.

IV.2. Signal To Noise Ratio At Detector

The diagonal elements of $\mathbf{W} \mathbf{R}_{e e} \mathbf{W}^{\dagger}$ are positive. Denote these as $d_{i}^{2}=\left(\mathbf{W} \mathbf{R}_{e e} \mathbf{W}^{\dagger}\right)_{i i}$. Then

$$
\sigma_{g}^{2}=\frac{1}{M} \sum_{i=0}^{M-1} \lambda_{i} d_{i}^{2}=\frac{1}{M} \sum_{i=0}^{M-1} d_{i}^{2} /\left|C[i] p_{i}\right|^{2}
$$

The signal to noise ratio at the detector is therefore

$$
\mathrm{SNR} \text { at detector }=\frac{\sigma_{s}^{2}}{\frac{1}{M} \sum_{i=0}^{M-1} d_{i}^{2} /\left|C[i] p_{i}\right|^{2}}
$$

IV.3. Channel Input Power

We can estimate the channel input power by referring to Fig. 5(b) which shows the unblocked version of the system. With $s(n)$ assumed to be white with variance $\sigma_{s}^{2}$, the autocorrelation of $\mathbf{v}(n)$ is

$$
\sigma_{s}^{2} \mathbf{W}^{-1} \boldsymbol{\Lambda}_{p} \mathbf{U}^{\dagger} \mathbf{U} \boldsymbol{\Lambda}_{p}^{\dagger} \mathbf{W}^{-\dagger}=\sigma_{s}^{2} \mathbf{W}^{-1} \boldsymbol{\Lambda}_{p} \boldsymbol{\Lambda}_{p}^{\dagger} \mathbf{W}^{-\dagger}
$$

Assuming that the cyclic prefix length is $L<<M$ we can approximate the channel input power as

$$
P_{i n} \approx \frac{\sigma_{s}^{2}}{M} \operatorname{Tr}\left(\mathbf{W}^{-1} \boldsymbol{\Lambda}_{p} \boldsymbol{\Lambda}_{p}^{\dagger} \mathbf{W}^{-\dagger}\right)=\frac{\sigma_{s}^{2}}{M^{2}} \sum_{i=0}^{M-1}\left|p_{i}\right|^{2}
$$

We will take the power constraint to be

$$
\sum_{i=0}^{M-1}\left|p_{i}\right|^{2}=M^{2} \quad \text { (power constraint) }
$$

so that the channel input power is $\sigma_{s}^{2}$.

\section{OPTIMIZING SNR}

It remains to optimize the SNR (4) under the power constraint (6). This is a standard problem which can be solved using a Lagrange variable, and the result is

$$
p_{i}=M \sqrt{\left|d_{i} / C[i]\right|} /\left(\sum_{k=0}^{M-1}\left|d_{k} / C[k]\right|\right)^{0.5}
$$

Substituting into Eq. (4) we obtain

$$
\text { optimized detector-SNR }=\frac{M^{3} \sigma_{s}^{2}}{\left(\sum_{k=0}^{M-1}\left|d_{k} / C[k]\right|\right)^{2}}
$$

If $e(n)$ is white with variance $\sigma_{e}^{2}$, then $\mathbf{R}_{e e}=\sigma_{e}^{2} \mathbf{I}$ and

$$
d_{k}^{2}=\left(\mathbf{W R}_{e e} \mathbf{W}^{\dagger}\right)_{k k}=M \sigma_{e}^{2}
$$


for all $k$. Then

$$
\text { optimized SNR at detector }=\frac{M^{2} \sigma_{s}^{2}}{\sigma_{e}^{2}\left(\sum_{k=0}^{M-1}\left|\frac{1}{C[k]}\right|\right)^{2}}
$$

We compare this system with the traditional zero-forcing equalizer shown in Fig. 6. With $S_{e e}\left(e^{j \omega}\right)$ denoting the power spectrum of $e(n)$, the signal to noise ratio is:

$$
\operatorname{SNR}(\text { traditional })=\frac{\sigma_{s}^{2}}{\int_{0}^{2 \pi} S_{e e}\left(e^{j \omega}\right)\left|1 / C\left(e^{j \omega}\right)\right|^{2} \frac{d \omega}{2 \pi}}
$$

Comparing with Eq. (7) we see that the improvement is

$$
\mathcal{G}_{\text {opt }}=\frac{M^{3} \int_{0}^{2 \pi} S_{e e}\left(e^{j \omega}\right)\left|1 / C\left(e^{j \omega}\right)\right|^{2} \frac{d \omega}{2 \pi}}{\left(\sum_{k=0}^{M-1}\left|d_{k} / C[k]\right|\right)^{2}}
$$

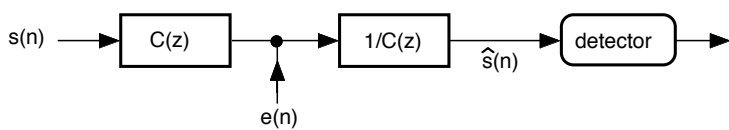

Figure 6. The ideal zero-forcing equalizer.

For large $M$ we can approximate the integral above with the sample average; the result is

$$
\mathcal{G}_{\text {opt }}=\frac{M^{2} \sum_{k=0}^{M-1} S_{e e}[k] /|C[k]|^{2}}{\left(\sum_{k=0}^{M-1}\left|d_{k} / C[k]\right|\right)^{2}}
$$

where $S_{e e}[k]=S_{e e}\left(e^{j 2 \pi k / M}\right)$. Recall that $d_{k}^{2}$ is given by (8). Using the relation between Toeplitz matrices and circulants for large $M$, we can make the approximation [2] $d_{k}^{2}=M S_{e e}[k]$. This yields

$$
\mathcal{G}_{o p t} \approx \frac{M \sum_{k=0}^{M-1} S_{e e}[k] /|C[k]|^{2}}{\left(\sum_{k=0}^{M-1} \sqrt{S_{e e}[k]} /|C[k]|\right)^{2}}
$$

Usign Cauchy-Schwartz inequality it follows that $\mathcal{G}_{\text {opt }} \geq 1$ with equality iff $S_{e e}[k] /|C[k]|^{2}$ is constant for all $k$.

\section{EXAMPLES AND CONCLUDING REMARKS}

Consider a channel with order $L=8$ and coefficients $c(n)$ given by $1.0000,-0.1379,0.7222,0.7183,0.2759$, $0.0423,0.3647,-0.0877$, and 0.0631 . All the zeros are inside the unit circle but there is a complex conjugate pair with radius close to unity $(=0.9755)$. The channel noise $e(n)$ was assumed to have autocorrelation $\sigma_{e}^{2}(0.3)^{|k|}$. We assume that $s(n)$ is an iid sequence coming from a 64-QAM constellation. Figure 7 shows the scatter diagram at the receiver, assuming $M=256$. Results for the traditional equalizer (Fig. 6) and equalizer with optimized $p_{k}$ (Sec. $\mathrm{V}$ ) are shown. The SNR at the channel output was fixed as $33 \mathrm{~dB}$ for both cases. The probabilities of error in Fig. 7 are 0.05 (top) and $7.8 \times 10^{-6}$ (bottom). In this example the optimized gain was $\mathcal{G}_{\text {opt }}=4.9282$. In this example, if we use $p_{k}=$ constant for all $k$, then the cyclic prefix system and the traditional equalizer Fig. 6 have nearly identical receiver SNR. Thus all the improvement comes because of optimization of $p_{k}$. As explained in Sec. III the cyclic prefix system has advantages over the traditional equalizer (even when all $p_{k}$ are identical) if $C(z)$ has some zeros outside the unit circle.

In conclusion, it appears that the cyclic prefix system can have broader applications in channel equalization. Note that the power allocation (optimization of $p_{k}$ ) is very different from the water pouring solution in DMT systems for bit rate maximization [3].
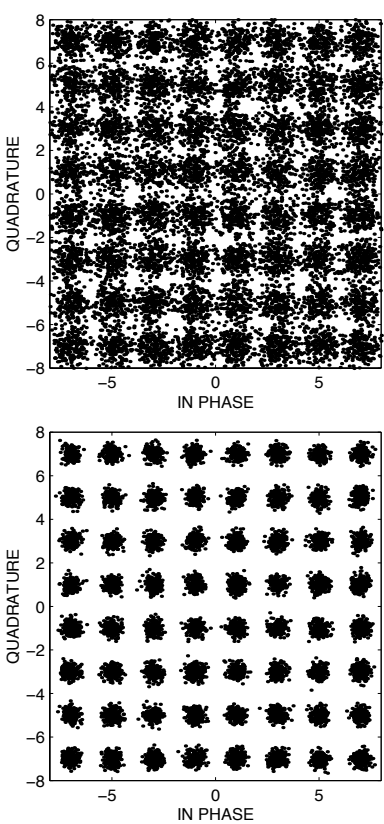

Figure 7. Results of equalization. Top: ideal zero forcing equalizer (Fig. 6). Bottom: optimal multipliers $\left\{p_{k}\right\}$.

\section{REFERENCES}

[1] Bingham, J. A. C. "Multicarrier modulation for data transmission: an idea whose time has come," IEEE Comm. Mag., pp. 5-14, May 1990.

[2] Gray, R. M. "On the asymptotic eigenvalue distribution of Toeplitz matrices," IEEE Trans. Info. Th., Nov. 1972.

[3] Kalet, I. "The multitone channel", IEEE Trans. Comm., pp. 119-124, Feb. 1989.

[4] Melsa, J. W., Younce, R. C., Rohrs, C. E., "Impulse response shortening for discrete multitone receivers," IEEE Trans. Comm, Dec. 1996.

[5] Mullis, C. T., and Roberts, R. A. "Synthesis of minimum roundoff noise fixed point digital filters," IEEE Trans. Circuits and Systems, , pp. 551-562, Sept. 1976.

[6] A. Papoulis, Signal analysis, McGraw Hill, 1977.

[7] Peled, A., and Ruiz, A. "Frequency domain data transmission using reduced computational complexity algorithms," IEEE ICASSP, pp. 964-967, Denver, CO, Apr. 1980.

[8] Starr, T., Cioffi, J. M., and Silverman, P. J. Understanding DSL technology, Prentice Hall, Inc., 1999. 